Homology, Homotopy and Applications, vol.16(2), 2014, pp.65-84

\title{
WEAK LEFSCHETZ FOR CHOW GROUPS: INFINITESIMAL LIFTING
}

\author{
D. PATEL AND G. V. RAVINDRA
}

(communicated by Charles A. Weibel)

\begin{abstract}
Let $X$ be a smooth projective variety over an algebraically closed field $k$ of characteristic zero, and let $Y \subset X$ be a smooth ample hyperplane section. The Weak Lefschetz conjecture for Chow groups states that the natural restriction map $\mathrm{CH}^{p}(X)_{\mathbb{Q}} \rightarrow \mathrm{CH}^{p}(Y)_{\mathbb{Q}}$ is an isomorphism for all $p<\operatorname{dim}(Y) / 2$. In this note, we revisit a strategy introduced by Grothendieck to attack this problem by using the Bloch-Quillen formula to factor this morphism through a continuous K-cohomology group on the formal completion of $X$ along $Y$. This splits the conjecture into two smaller conjectures: one consisting of an algebraization problem and the other dealing with infinitesimal liftings of algebraic cycles. We give a complete proof of the infinitesimal part of the conjecture.
\end{abstract}

\section{Introduction}

In this note, we continue our study (see [13]) of weak Lefschetz-type theorems for Chow groups modulo rational equivalence. We begin by recalling the weak Lefschetz conjecture for Chow groups. In the following, let $X$ be a smooth projective variety over an algebraically closed field $k$ of characteristic zero. Furthermore, let $Y \subset X$ denote a smooth ample hyperplane section.

Conjecture 1.1. The natural restriction map

$$
\mathrm{CH}^{p}(X)_{\mathbb{Q}} \longrightarrow \mathrm{CH}^{p}(Y)_{\mathbb{Q}}
$$

is an isomorphism for all $p<\operatorname{dim}(Y) / 2$.

When $p=1$, the Chow group of divisors of any smooth, projective variety can be identified with the Picard group, and the above conjecture is the GrothendieckLefschetz theorem, which, in fact, holds integrally. Grothendieck's proof (see [8]) for Picard groups proceeded by first lifting line bundles from $Y$ to the formal completion

The first author was supported by the ANR program $p$-adic Hodge theory and beyond (ThHopaD) ANR-11-BS01-005. The second author was partially supported by grant \# 207893 from the Simons Foundation.

Received April 9, 2014, revised April 28, 2014; published on July 21, 2014.

2010 Mathematics Subject Classification: 14C25, 14C35.

Key words and phrases: K-theory, algebraic cycles.

Article available at http://dx.doi.org/10.4310/HHA.2014.v16.n2.a4

Copyright (C) 2014, International Press. Permission to copy for private use granted. 
$\mathfrak{X}$ of $X$ along $Y$, and then to make use of the Lefschetz conditions to extend line bundles from the formal scheme to the whole scheme $X$. Unfortunately, this strategy does not directly apply to higher codimension cycles, since the Chow groups are invariant under infinitesimal thickenings. However, the Bloch-Quillen formula allows one to interpret the Chow groups in terms of K-cohomology, where one can try to apply deformation theoretic methods. We briefly recall the formalism.

Let $\mathcal{K}_{i, X}$ denote the $i$ th K-theory sheaf in the Zariski topology (cf. $\S 2.3$ ). Then the Bloch-Quillen formula gives an isomorphism

$$
\mathrm{H}^{p}\left(X, \mathcal{K}_{p, X}\right) \stackrel{\cong}{\longrightarrow} \mathrm{CH}^{p}(X) .
$$

One has a similar statement for $Y$. The restriction morphism from the conjecture is then the restriction map

$$
\mathrm{H}^{p}\left(X, \mathcal{K}_{p, X}\right)_{\mathbb{Q}} \longrightarrow \mathrm{H}^{p}\left(Y, \mathcal{K}_{p, Y}\right)_{\mathbb{Q}}
$$

and the conjecture is equivalent to showing that this map is an isomorphism for all $p<\operatorname{dim}(Y) / 2$. Let $Y_{n}$ denote the $n$th infinitesimal neighborhood of $Y$ in $X$. Then we can consider $\left(\mathcal{K}_{i, Y_{n}}\right)$ as a pro-sheaf on $Y$ (cf. $\left.\S 2.3\right)$, and, in particular, consider its continuous cohomology (see $[\mathbf{1 0}]$ ). Then one can factor the above morphism (see $[\mathbf{1 3}$, $\S 3])$ as a composition:

$$
\mathrm{H}^{p}\left(X, \mathcal{K}_{p, X}\right)_{\mathbb{Q}} \longrightarrow \mathrm{H}_{\text {cont }}^{p}\left(\mathfrak{X},\left(\mathcal{K}_{p, Y_{n}}\right)\right)_{\mathbb{Q}} \longrightarrow \mathrm{H}^{p}\left(Y, \mathcal{K}_{p, Y}\right)_{\mathbb{Q}}
$$

Therefore, the following two conjectures imply Conjecture 1.1:

Conjecture 1.2. The natural morphism

$$
\mathrm{H}^{p}\left(X, \mathcal{K}_{p, X}\right)_{\mathbb{Q}} \longrightarrow \mathrm{H}_{\text {cont }}^{p}\left(\mathfrak{X},\left(\mathcal{K}_{p, Y_{n}}\right)\right)_{\mathbb{Q}}
$$

is an isomorphism for all $p<\operatorname{dim}(Y) / 2$.

Conjecture 1.3. The natural morphism

$$
\mathrm{H}_{\text {cont }}^{p}\left(\mathfrak{X},\left(\mathcal{K}_{p, Y_{n}}\right)\right)_{\mathbb{Q}} \longrightarrow \mathrm{H}^{p}\left(Y, \mathcal{K}_{p, Y}\right)_{\mathbb{Q}}
$$

is an isomorphism for all $p<\operatorname{dim}(Y) / 2$.

In [13], the authors proved conjecture 1.3 for $p=2$. In this note, we give a complete proof of this conjecture. In particular, we prove the following theorem.

Theorem 1.4. Let $X$ be a smooth projective variety over an algebraically closed field $k$ of characteristic zero and $Y$ a smooth ample hyperplane section. Then the natural restriction map

$$
\mathrm{H}_{\text {cont }}^{p}\left(\mathfrak{X},\left(\mathcal{K}_{q, Y_{n}}\right)\right) \longrightarrow \mathrm{H}^{p}\left(Y, \mathcal{K}_{q, Y}\right)
$$

is an isomorphism for all $p+q<\operatorname{dim}(Y)$. In particular,

$$
\mathrm{H}_{\text {cont }}^{p}\left(\mathfrak{X},\left(\mathcal{K}_{p, Y_{n}}\right)\right) \longrightarrow \mathrm{H}^{p}\left(Y, \mathcal{K}_{p, Y}\right)
$$

is an isomorphism for all $p<\operatorname{dim}(Y) / 2$.

Note that the above theorem does not require one to tensor with $\mathbb{Q}$. The basic strategy to prove the above conjecture in the case of $p=2$ was to use Bloch's theorem 
(see $[\mathbf{2}]$ ) describing the kernel of the (surjective) morphism

$$
\mathcal{K}_{2, Y_{n}} \longrightarrow \mathcal{K}_{2, Y}
$$

in terms of 1-forms. The proof of Theorem 1.4 proceeds in a similar manner. Instead of Bloch's theorem, we make use of Goodwillie's theorem, which relates the kernel of the morphism

$$
\mathcal{K}_{p, Y_{n}} \longrightarrow \mathcal{K}_{p, Y}
$$

to cyclic homology (cf. §2.3). In particular, Goodwillie's theorem gives an isomorphism of this kernel with the kernel of the corresponding morphism at the level of cyclic homology sheaves over $\mathbb{Q}$

$$
\mathcal{H C}_{p-1, Y_{n}}^{/ \mathbb{Q}} \longrightarrow \mathcal{H C}_{p-1, Y}^{/ \mathbb{Q}}
$$

Therefore, the bulk of the proof goes into computing the cohomology of these sheaves. Since $Y$ is smooth, its cyclic homology sheaves are well understood by the classical Hochschild-Kostant-Rosenberg isomorphism. On the other hand, the cyclic homology sheaves for $Y_{n}$ are not easy to understand. However, if one assembles all of these together into a pro-sheaf, then one has a pro-analog of the classical HochschildKostant-Rosenberg isomorphism [4]. This reduces the proof of the theorem to some standard computations with (pro) de Rham complexes.

In this note, we say nothing about conjecture 1.2, which is clearly the more difficult conjecture. The morphism

$$
\mathrm{H}^{p}\left(X, \mathcal{K}_{p, X}\right) \longrightarrow \mathrm{H}_{\text {cont }}^{p}\left(\mathfrak{X},\left(\mathcal{K}_{p, Y_{n}}\right)\right)
$$

factors as (see $[\mathbf{1 3}, \S 3])$

$$
\mathrm{H}^{p}\left(X, \mathcal{K}_{p, X}\right) \longrightarrow \mathrm{H}^{p}\left(\mathfrak{X}, \mathcal{K}_{p, \mathfrak{X}}\right) \longrightarrow \mathrm{H}_{\text {cont }}^{p}\left(\mathfrak{X},\left(\mathcal{K}_{p, Y_{n}}\right)\right) .
$$

The second arrow above can again be understood via cyclic homology (when $p=1$, this is an isomorphism, see [13, Proposition 3.1], and we hope to pursue this elsewhere. On the other hand, it is not clear how to analyze the first arrow. Note that one has a global analog of the above picture. Namely, there is a sequence of morphisms of K-theory spectra:

$$
\mathrm{K}(X) \longrightarrow \mathrm{K}(\mathfrak{X}) \longrightarrow \mathrm{K}^{\text {top }}(\mathfrak{X}) \longrightarrow \mathrm{K}(Y) .
$$

Here $\mathrm{K}^{t o p}(\mathfrak{X})$ is by definition the homotopy inverse limit of the spectra $\mathrm{K}\left(Y_{n}\right)$. This gives a diagram of global K-theory groups:

$$
\mathrm{K}_{p}(X) \longrightarrow \mathrm{K}_{p}(\mathfrak{X}) \longrightarrow \mathrm{K}_{p}^{\text {top }}(\mathfrak{X}) \longrightarrow \mathrm{K}_{p}(Y) .
$$

Furthermore, in the case of $\mathrm{K}(X)$ and $\mathrm{K}(Y)$, the Brown-Gersten-Quillen (BGQ) spectral sequence allows one to relate the $\mathrm{K}$-cohomology groups with the global $\mathrm{K}$ theory groups. While there is an analog of the BGQ-spectral sequence for $\mathrm{K}^{\text {top }}(\mathfrak{X})$, with $\mathrm{E}_{2}^{p, q}$ terms given by the continuous cohomology groups above, there seems to be no such spectral sequence for $\mathrm{K}_{p}(\mathfrak{X})$. The obstruction to the existence of such a spectral sequence seems to be due to the lack of Zariski descent in this case. The interested reader may refer to $[\mathbf{3}]$ and $[\mathbf{7}]$ for related work in a different setting.

We conclude this introduction with a brief description of the contents of each section. In $\S 2$, we recall some preliminaries on pro-systems, K-theory, and Hochschild homology (and its various other cousins). In $\S 2.1$, we recall some statements from the 
theory of pro-sheaves. In $\S 2.2$, we recall Hochschild homology and its cousins, and, in particular, the pro-HKR isomorphism adapted to our setting of pro-sheaves. In $\S 2.3$, after some preliminaries on K-theory, we recall Goodwillie's theorem relating relative K-theory along infinitesimal extensions to negative cyclic homology. In $\S 2.4$, we recall the Kassel-Sletsjøe spectral sequence (adapted to our setting of pro-sheaves), which relates Hochschild homology over a base field $k$ with the Hochschild homology over a subfield $F$ of $k$. In $\S 3$, we compare continuous cohomology of cyclic homology prosheaves on $\mathfrak{X}$ with that of the cohomology of cyclic cohomology sheaves on $Y$. The results of $\S 2.2$ reduce this to a computation of various sheaves of differentials and de Rham cohomology sheaves. In $\S 3.1$, we recall a result of Ogus, which compares the pro-system of de Rham cohomology sheaves on $\mathfrak{X}$ and $Y$. In $\S 3.2$, we use Ogus's result and some basic results from algebraic geometry (Kodaira-Nakano vanishing) to prove isomorphisms between the continuous cohomology of pro-sheaves of differentials on $\mathfrak{X}$ with the cohomology of sheaves of differentials on $Y$. In $\S 3.3$, the results of $\S 3.2$ are extended, using the Kassel-Sletsjøe spectral sequence, to an arbitrary subfield $F \subset k$. Finally, in $\S 4$ we give a proof of the main result.

\section{Preliminaries}

\subsection{Preliminaries on pro-systems}

For any abelian category $\mathcal{A}$, let $\operatorname{Pro}(\mathcal{A})$ denote the category of pro-objects in $\mathcal{A}$. Let $\mathcal{A}^{\mathbb{N}}$ denote the full subcategory of the category of pro-objects consisting of proobjects indexed by $\mathbb{N}$. We shall use the notation $\left(A_{n}\right)$ to denote an object in this category. Note that any object $A \in \mathcal{A}$ gives rise to a constant pro-system denoted $(A)$. We shall sometimes denote this simply by $A$. Recall that morphisms between two objects $\left(A_{n}\right)$ and $\left(B_{n}\right)$ in this category are given by

$$
\operatorname{Hom}_{\mathcal{A}^{\mathbb{N}}}\left(\left(A_{n}\right),\left(B_{n}\right)\right)=\underset{\longleftrightarrow}{\lim } \underset{\lim _{H}}{\longrightarrow} \operatorname{mom}_{\mathcal{A}}\left(A_{i}, B_{j}\right) \text {. }
$$

In particular, a system of morphisms $f_{n}: A_{n} \rightarrow B_{n}$ compatible with the transition maps $A_{n} \rightarrow A_{n-1}$ and $B_{n} \rightarrow B_{n-1}$ gives rise to a morphism of pro-systems. We shall refer to such morphisms as strict morphisms. In the following, we shall also be interested in $C h\left(\mathcal{A}^{\mathbb{N}}\right)$, the corresponding category of (co) chain complexes of pro-systems. Note that both $\mathcal{A}^{\mathbb{N}}$ and $C h\left(\mathcal{A}^{\mathbb{N}}\right)$ are abelian categories. In the following, by proobjects in $\mathcal{A}$ we will always mean objects in $\mathcal{A}^{\mathbb{N}}$. We refer the reader to [1, Appendix] for details regarding pro-objects.

Suppose that $\mathcal{A}$ is the category of sheaves of abelian groups on some topological space $X$. In this situation, the corresponding category of pro-sheaves has enough injectives. In particular, following Jannsen $[\mathbf{1 0}]$, we denote by $\mathrm{H}_{\text {cont }}^{p}\left(X,\left(\mathcal{F}_{n}\right)\right)$ the continuous cohomology groups of the given pro-system. In this setting, one has the following standard exact sequence:

$$
0 \longrightarrow R^{1} \varliminf_{\mathfrak{i m}} H^{p-1}\left(X, \mathcal{F}_{n}\right) \longrightarrow \mathrm{H}_{\text {cont }}^{p}\left(X,\left(\mathcal{F}_{n}\right)\right) \longrightarrow \lim ^{p}\left(X, \mathcal{F}_{n}\right) \longrightarrow 0 .
$$

If

$$
0 \longrightarrow\left(\mathcal{F}_{n}^{\prime}\right) \longrightarrow\left(\mathcal{F}_{n}\right) \longrightarrow\left(\mathcal{F}_{n}^{\prime \prime}\right) \longrightarrow 0
$$

is a complex of strict morphisms, then it is exact if

$$
0 \longrightarrow \mathcal{F}_{n}^{\prime} \longrightarrow \mathcal{F}_{n} \longrightarrow \mathcal{F}_{n}^{\prime \prime} \longrightarrow 0
$$


is exact for all $n$. This follows from the fact that the kernel of a strict morphism $\left(f_{n}\right):\left(\mathcal{F}_{n}\right) \rightarrow\left(\mathcal{G}_{n}\right)$ is given by the pro-system $\left(k e r\left(f_{n}\right)\right)$, and similarly for the cokernel.

\subsection{Preliminaries on cyclic homology}

In this section, we recall some background material on various non-commutative homology theories. We refer to [16, Chapter 9] for details. In the following, $k$ will denote a fixed field of characteristic zero.

A mixed complex over $k$ is a triple $(C, b, B)$ where $(C \cdot b)$ is a chain complex of $k$-vector spaces and $B: C^{\cdot} \rightarrow C^{\cdot}[1]$ is a morphism of degree 1 such that $B^{2}=0$.

Example 2.1. Given a commutative ring $A$ over $k$, we can consider the mixed complex $\left(\Omega_{A / k}, 0, d\right)$. In particular, we put $\Omega_{A / k}^{p}$ in degree $p, b=0$ and $B=d$.

Given a mixed complex $\left(C^{\cdot}, b, B\right)$, one can associate to it the cyclic homology chain complex $\mathrm{HC}\left(C^{\cdot}\right)$ whose homology groups $\mathrm{HC}_{i}\left(C^{\cdot}\right)$ are called the cyclic homology groups. One can also associate to it the negative cyclic (resp. periodic cyclic) homology $\mathrm{HN}\left(C^{\cdot}\right)$ (resp. $\mathrm{HP}\left(C^{\cdot}\right)$ ) chain complex, whose homology groups are the negative cyclic (resp. periodic cyclic) homology groups. Furthermore, the homology groups of the underlying complex $\left(C^{\cdot}, b\right)$ will be referred to as the Hochschild homology groups. These chain complexes are related by the following two standard exact sequences of chain complexes:

1. $0 \longrightarrow \mathrm{C}^{\cdot} \longrightarrow \mathrm{HC}\left(C^{\cdot}\right) \longrightarrow \mathrm{HC}\left(C^{\cdot}\right)[2] \longrightarrow 0$.

2. $0 \longrightarrow \mathrm{HN}\left(C^{\cdot}\right) \longrightarrow \mathrm{HP}\left(C^{\cdot}\right) \longrightarrow \mathrm{HC}\left(C^{\cdot}\right)[2] \longrightarrow 0$.

A morphism of mixed complexes $f:\left(C^{\prime}, b, B\right) \rightarrow\left(C^{\prime}, b^{\prime}, B^{\prime}\right)$ is said to be a quasiisomorphism if it induces a quasi-isomorphism of underlying complexes $C^{\cdot} \rightarrow C^{\prime}$. A quasi-isomorphism of mixed complexes induces an isomorphism on the corresponding Hochschild and cyclic homology groups [16, p. 348].

Given a commutative ring $A$ over $k$, there is a standard mixed complex $\left(C^{\cdot}(A), b, B\right)$ associated to $A$. The corresponding Hochshild (respectively cyclic, negative cyclic, and periodic cyclic) homology groups will be denoted by $\mathrm{HH}_{q}^{/ k}(A)$ (respectively $\mathrm{HC}_{q}^{/ k}(A)$, $\mathrm{HN}_{q}^{/ k}(A)$, and $\left.\operatorname{HP}_{q}^{/ k}(A)\right)$.

Let $X$ be a scheme over $k$. Then one can sheafify the constructions of the previous paragraph. In particular, one can associate to $X$ a mixed complex of sheaves $(\mathcal{C} \cdot(X), b, B)$. One has the corresponding Hochschild (respectively periodic cyclic, negative cyclic, and cyclic) homology sheaves $\mathcal{H H}_{q, X}^{/ k}\left(\right.$ resp. $\left.\mathcal{H P}_{q, X}^{/ k}, \mathcal{H N}_{q, X}^{/ k}, \mathcal{H C}_{q, X}^{/ k}\right)$ over $X$.

If $A$ is a commutative algebra over a field $k$ of characteristic zero, then one has a natural morphism (antisymmetrization map) of graded $A$-modules:

$$
\Omega_{A / k}^{q} \longrightarrow \mathrm{HH}_{q}^{/ k}(A) \text {. }
$$

Furthermore, if $A$ is a (noetherian) regular algebra over $k$, then the above morphism is an isomorphism. On the other hand, one has a natural morphism of mixed complexes

$$
\mu:\left(C^{\cdot}(A), b, B\right) \longrightarrow\left(\Omega_{A / k}, 0, d\right),
$$

which, at the level of homology, is multiplication by $q+1$ in degree $q$ when precomposed with

$$
\Omega_{A / k}^{q} \longrightarrow \mathrm{HH}_{q}^{/ k}(A) .
$$


It follows that if $A$ is regular, then $\mu$ is a quasi-isomorphism of mixed complexes. In this setting, one has the following description for the cyclic homology of $A$ over $k$ :

$$
\mathrm{HC}_{q}^{/ k}(A)=\Omega_{A / k}^{q} / d \Omega_{A / k}^{q-1} \oplus \mathrm{H}_{d R}^{q-2}(A) \oplus \mathrm{H}_{d R}^{q-4}(A) \oplus \cdots .
$$

Here $\mathrm{H}_{d R}^{q}(A)$ is the cohomology of the de Rham complex $\left(\Omega_{A / k}, d\right)$ of $A$ over $k$. If $X$ is a smooth scheme over $k$, then we can sheafify these results. In particular, one has an induced isomorphism of sheaves

$$
\Omega_{X / k}^{q} \longrightarrow \mathcal{H H}_{q, X}^{/ k}
$$

Furthermore, the natural morphism of mixed complexes

$$
\mu:(\mathcal{C}(X), b, B) \longrightarrow\left(\Omega_{X / k}, 0, d\right)
$$

is a quasi-isomorphism. It follows that one has an isomorphism

$$
\mathcal{H C}_{q, X}^{/ k} \longrightarrow \Omega_{X / k}^{q} / d \Omega_{X / k}^{q-1} \oplus \mathcal{H}_{d R}^{q-2}(X / k) \oplus \mathcal{H}_{d R}^{q-4}(X / k) \oplus \cdots
$$

One can generalize the result of the previous paragraph to the pro-setting. We shall assume for the remainder of this section that $A$ is an algebra essentially of finite type and smooth over a field $k$ of characteristic zero and that $F$ is a subfield of $k$. Let $I \subset A$ be an ideal. Then one has the following theorem due to Cortiñas, Haesemeyer, and Weibel.

Theorem 2.2. ([4, Theorem 3.2, Proposition 3.5]) The induced morphism of graded pro-A-modules

$$
\left(\Omega_{\left(A / I^{m}\right) / F}^{q}\right) \longrightarrow\left(\mathrm{HH}_{q}^{/ F}\left(A / I^{m}\right)\right)
$$

is a pro-isomorphism.

Proof. This is precisely Theorem 3.2 of loc. cit. when $F=k$ and Proposition 3.5 for general $F$.

On the other hand, one has a morphism of pro-mixed complexes

$$
\mu:\left(\mathcal{C} \cdot\left(A / I^{m}\right), b, B\right) \longrightarrow\left(\Omega_{\left(A / I^{m}\right) / F}, 0, d\right) .
$$

Again, for each $m$, the composition

$$
\Omega_{\left(A / I^{m}\right) / F}^{q} \longrightarrow \mathrm{HH}_{q}^{/ F}\left(A / I^{m}\right) \longrightarrow \Omega_{\left(A / I^{m}\right) / F}^{q}
$$

is multiplication by $q+1$.

Lemma 2.3. In the above setting, $\mu$ is a quasi-isomorphism of pro-complexes.

Proof. We must show that the induced morphism

$$
\left(\mathrm{HH}_{q}^{/ F}\left(A / I^{m}\right)\right) \longrightarrow\left(\Omega_{\left(A / I^{m}\right) / F}^{p}\right)
$$

is an isomorphism of graded pro-modules. By the previous remarks we have that the composition

$$
\left(\Omega_{\left(A / I^{m}\right) / F}^{q}\right) \longrightarrow\left(\mathrm{HH}_{q}^{/ F}\left(A / I^{m}\right)\right) \longrightarrow\left(\Omega_{\left(A / I^{m}\right) / F}^{q}\right)
$$

is multiplication by $q+1$, and the first arrow is an isomorphism of pro-modules. Since $F \supset \mathbb{Q}$, it follows that the right morphism divided by $q+1$ is an inverse of the first morphism. Therefore, the right arrow is an isomorphism. 
Corollary 2.4. One has an isomorphism of pro-modules

$$
\left(\mathrm{HC}_{q}^{/ F}\left(A / I^{m}\right)\right) \longrightarrow\left(\Omega_{\left(A / I^{m}\right) / F}^{q} / d \Omega_{\left(A / I^{m}\right) / F}^{q-1}\right) \oplus\left(\mathrm{H}_{d R}^{q-2}\left(\left(A / I^{m}\right) / F\right)\right) \oplus \cdots .
$$

Suppose now that $X$ is a smooth scheme essentially of finite type over a field $k$ of characteristic zero. Let $Y \subset X$ denote a closed smooth subvariety of $X$, and let $Y_{n}$ denote the $n$th infinitesimal thickening of $Y$ in $X$. In this situation, we can sheafify the previous constructions to get a morphism of mixed complexes of pro-sheaves

$$
\mu:\left(\left(\mathcal{C} \cdot\left(Y_{n}\right), b, B\right)\right) \longrightarrow\left(\left(\Omega_{Y_{n / k}}, 0, d\right)\right) .
$$

Lemma 2.5. Let $X$ be as above. Then the natural antisymmetrization map

$$
\left(\Omega_{Y_{n} / k}^{q}\right) \longrightarrow\left(\mathcal{H H}_{q, Y_{n}}^{/ k}\right) .
$$

is a pro-isomorphism.

Proof. It is enough to check this locally on $X$. On the other hand, in the affine case this is precisely the theorem of Cortiñas, Haesaemeyer, and Weibel recalled above.

Corollary 2.6. Let $X$ be a smooth variety over a field $k$, and let $F \subset k$ be a subfield. Suppose $Y \subset X$ is a smooth closed subvariety and $Y_{n}$ is the nth infinitesimal thickening of $Y$ in $X$. Then one has an isomorphism of (graded) pro-sheaves on $Y$

$$
\left(\mathcal{H C}_{q, Y_{n}}^{/ F}\right) \longrightarrow\left(\Omega_{Y_{n} / F}^{q} / d \Omega_{Y_{n} / F}^{q-1}\right) \oplus\left(\mathcal{H}_{d R}^{q-2}\left(Y_{n} / F\right)\right) \oplus\left(\mathcal{H}_{d R}^{q-4}\left(Y_{n} / F\right)\right) \cdots
$$

Proof. It is enough to show that

$$
\mu:\left(\mathcal{C} \cdot\left(Y_{n}\right)\right) \longrightarrow\left(\Omega_{Y_{n} / F}\right) .
$$

is a quasi-isomorphism of mixed complexes of pro-sheaves. Again, this can be checked locally on $X$. In this case, it is precisely Lemma 2.3 above.

\subsection{Preliminaries on K-theory}

For any scheme $X$, we let $\mathrm{K}^{\text {perf }}(X)$ denote the (non-connective) K-theory spectrum of perfect complexes on $X$ and $\mathrm{K}_{q}^{\text {perf }}(X)$, the corresponding homotopy groups. It follows from [15], that if $X$ is a smooth scheme then the natural map $\mathrm{K}(X) \rightarrow$ $\mathrm{K}^{\text {perf }}(X)$ is a weak equivalence, where $\mathrm{K}(X)$ is the usual K-theory spectrum of vector bundles on $X$. In general, if $X$ has an ample family of line bundles then $\mathrm{K}_{i}(X)=$ $\mathrm{K}_{i}^{\text {perf }}(X)$ for all $i \geqslant 0$.

We denote by $\mathcal{K}_{X}$ the pre-sheaf of spectra on $X$ that associates to an open $U \subset X$ the spectrum $\mathrm{K}(U)$, and similarly for $\mathcal{K}^{\text {perf }}$. Then the corresponding homotopy sheaves are given by $\mathcal{K}_{i, X}$ and $\mathcal{K}_{i, X}^{\text {perf }}$. By definition, $\mathcal{K}_{i, X}$ is the sheaf associated to the pre-sheaf whose sections over $U \subset X$ are given by $\mathrm{K}_{i}(U)$, and similarly for $\mathcal{K}_{i, X}^{\text {perf }}$.

Remark 2.7. Strictly speaking, $\mathcal{K}_{X}^{\text {perf }}$ is the pre-sheaf that takes the DG-category of perfect complexes on $U$ to the spectrum $\mathrm{K}^{\text {perf }}(U)$. To avoid dealing with pseudofunctors, one should take some rectification of the corresponding (pseudo) pre-sheaf of DG-categories. We refer to [17] for details. 
Suppose $Y \subset X$ is a closed subvariety and $Y_{n}$ is its $n$th infinitesimal neighborhood. The we can consider $\mathcal{K}_{Y_{n}}^{\text {perf }}$ as pre-sheaves on $Y$. Let $\mathcal{K}_{\left(Y, Y_{n}\right)}^{\text {perf }}$ denote the homotopy fiber of

$$
\mathcal{K}_{Y_{n}}^{\text {perf }} \longrightarrow \mathcal{K}_{Y}^{\text {perf }}
$$

In particular, one has a long exact sequence of homotopy sheaves

$$
\cdots \longrightarrow \mathcal{K}_{i,\left(Y_{n}, Y\right)}^{\text {perf }} \longrightarrow \mathcal{K}_{i, Y_{n}}^{\text {perf }} \longrightarrow \mathcal{K}_{i, Y}^{\text {perf }} \longrightarrow \mathcal{K}_{i-1,\left(Y_{n}, Y\right)}^{\text {perf }} \longrightarrow \cdots
$$

where $\mathcal{K}_{i,\left(Y_{n}, Y\right)}^{\text {perf }}$ are the sheaves of homotopy groups associated to $\mathcal{K}_{\left(Y_{n}, Y\right)}^{\text {perf }}$.

Remark 2.8. In the following, we shall always assume that our (presheaves of) spectra and presheaves of spectra are fibrant-cofibrant. In particular, we choose once and for all a functorial fibrant-cofibrant replacement. ${ }^{1}$

There is a standard way to associate a pre-sheaf of spectra to a chain complex of sheaves such that the homotopy sheaves of the corresponding pre-sheaf of spectra are the homology sheaves of the given complex. Given a scheme $X / k$ and a subfield $F \subset k$, let $\mathcal{H H}_{X}^{/ F}$ denote the corresponding Hochschild homology pre-sheaf of spectra relative to $F$. Similarly, let $\mathcal{H} \mathcal{N}_{X}^{/ F}$ (resp. $\mathcal{H C}_{X}^{/ F}$ and $\mathcal{H} \mathcal{P}_{X}^{/ F}$ ) denote the corresponding negative cyclic (resp. cyclic and periodic cyclic) homology presheaves of spectra. Just as above, we let $\mathcal{H H}_{\left(Y_{n}, Y\right)}^{/ F}$ denote the homotopy fiber of the restriction map $\mathcal{H H}_{Y_{n}}^{/ F} \rightarrow \mathcal{H H}_{Y}^{/ F}$. One defines $\mathcal{H N}_{\left(Y_{n}, Y\right)}^{/ F}, \mathcal{H C}_{\left(Y_{n}, Y\right)}^{/ F}$, and $\mathcal{H P}_{\left(Y_{n}, Y\right)}^{/ F}$ in a similar manner. The short exact sequence

$$
0 \longrightarrow \mathrm{HN}\left(C^{\cdot}\right) \longrightarrow \mathrm{HP}\left(C^{\cdot}\right) \longrightarrow \mathrm{HC}\left(C^{\cdot}\right)[-2] \longrightarrow 0
$$

sheafifies to give a cofibre sequence of presheaves of spectra:

$$
\mathcal{H N}_{X}^{/ F} \longrightarrow \mathcal{H P}_{X}^{/ F} \longrightarrow \Omega^{-2} \mathcal{H C}_{X}^{/ F}
$$

For the inclusions $Y \hookrightarrow Y_{n}$, this gives rise to a commutative diagram of presheaves of spectra:

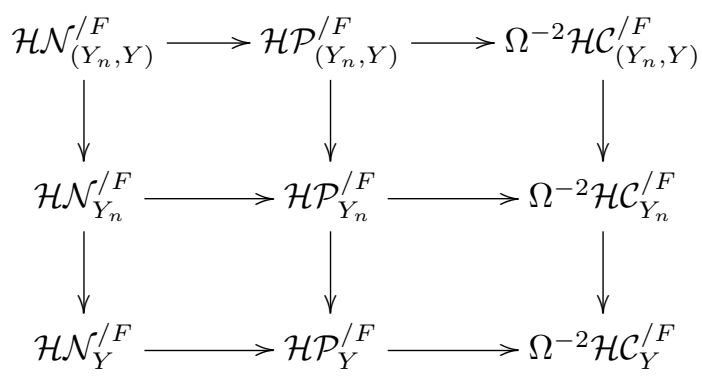

By a theorem of Goodwillie [5, Theorem II.5.1], $\mathcal{H P}$ is invariant under infinitesimal thickenings. In particular, the natural morphism

$$
\mathcal{H} \mathcal{P}_{Y_{n}}^{/ F} \longrightarrow \mathcal{H} \mathcal{P}_{Y}^{/ F}
$$

is a weak equivalence. It follows that $\mathcal{H P}_{\left(Y_{n}, Y\right)}^{/ F}$ is contractible, and, in particular, the

${ }^{1}$ In this note, we work with the local projective model structure on the category of pre-sheaves of spectra. 
corresponding homotopy sheaves are trivial. It follows that one has an isomorphism of sheaves

$$
\mathcal{H C}_{i-1,\left(Y_{n}, Y\right)}^{/ F} \longrightarrow \mathcal{H N}_{i,\left(Y_{n}, Y\right)}^{/ F}
$$

Suppose now $X$ is a scheme over a field $k$ of characteristic zero. Let $Y \subset X$ denote a closed subvariety, and let $Y_{n}$ denote its $n$th infinitesimal thickening. In this situation, Goodwillie's theorem [6, Theorem 4.5] allows one to identify the sheaves $\mathcal{K}_{\left(Y_{n}, Y\right)}^{\text {perf }}$ with negative cyclic homology. We shall use this result in the following to reduce the computation of relative $K$-theory to that of relative negative cyclic homology. There is a natural chern character

$$
\mathrm{K}_{X}^{\text {perf }} \rightarrow \mathcal{H} \mathcal{N}_{X}^{/ \mathbb{Q}}
$$

This gives rise to a diagram of presheaves of spectra:

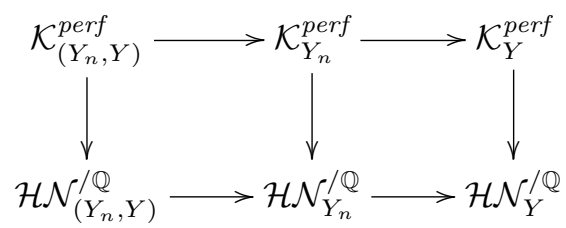

Goodwillie's theorem says that the left vertical arrow is a weak equivalence. In particular, it induces an isomorphism on the corresponding sheaves of homotopy groups. Combining the results of the previous paragraph, one has an isomorphism

$$
\mathcal{K}_{i,\left(Y_{n}, Y\right)} \longrightarrow \mathcal{H C}_{i-1,\left(Y_{n}, Y\right)}^{/ \mathbb{Q}} .
$$

We now recall a pro-sheaf version of the previous results. For the rest of this section, we assume that $X$ is a smooth variety over a field $k$ of characteristic zero and $Y$ is a closed smooth subvariety. As before, $Y_{n}$ is the $n$th infinitesimal neighborhood of $Y$ in $X$. By [13, Lemma 5.9], the natural restriction maps

$$
\mathcal{K}_{i, Y_{n}}^{\text {perf }} \longrightarrow \mathcal{K}_{i, Y}^{\text {perf }}
$$

and

$$
\mathcal{H C}_{i, Y_{n}}^{/ F} \longrightarrow \mathcal{H C}_{i, Y}^{/ F}
$$

are surjective. It follows that we have short exact sequences

$$
0 \longrightarrow \mathcal{K}_{i,\left(Y_{n}, Y\right)}^{\text {perf }} \longrightarrow \mathcal{K}_{i, Y_{n}}^{\text {perf }} \longrightarrow \mathcal{K}_{i, Y}^{\text {perf }} \longrightarrow 0,
$$

and

$$
0 \longrightarrow \mathcal{H C}_{i,\left(Y_{n}, Y\right)}^{/ F} \longrightarrow \mathcal{H C}_{i, Y_{n}}^{/ F} \longrightarrow \mathcal{H C}_{i, Y}^{/ F} \longrightarrow 0 .
$$

This gives rise to a commutative diagram of pro-sheaves with exact rows:

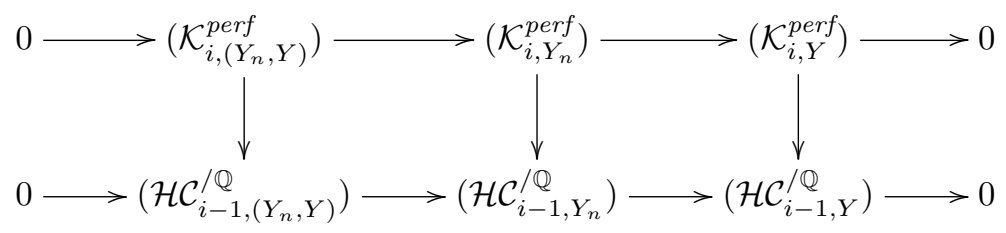

We record the following corollary for future reference: 
Corollary 2.9. Let $X$ and $Y$ be as above. Then, by (1), one has an isomorphism

$$
\mathrm{H}_{\text {cont }}^{p}\left(Y,\left(\mathcal{K}_{q,\left(Y_{n}, Y\right)}^{\text {perf }}\right)\right) \longrightarrow \mathrm{H}_{\text {cont }}^{p}\left(Y,\left(\mathcal{H C}_{q-1,\left(Y_{n}, Y\right)}^{/ \mathbb{Q}}\right)\right)
$$

\subsection{Pro-Kassel-Sletsjøe spectral sequence}

In the following, we will need to compute continuous cohomology groups on a scheme $Y / k$ with coefficients given by the pro-system $\left(\mathcal{H H}_{p, Y_{n}}^{/ \mathbb{Q}}\right)$ (with notation as in the previous section). We shall achieve this by first using geometric methods to compute the analogous result over $k$ and then descending to $\mathbb{Q}$. The Kassel-Sletsjøe spectral sequence ([11, 4.3a]; [4, Lemma 3.4]) allows one to pass from $k$ to a subfield $F$. We recall the relevant statements in our setting of pro-sheaves.

Given a commutative algebra $A$ over $k$, one has a natural decomposition for Hochschild homology:

$$
\mathrm{HH}_{p}^{/ k}(A) \cong \oplus \mathrm{HH}_{p}^{(i), / k}(A) .
$$

Furthermore, one has $\operatorname{HH}_{p}^{(p), / k}(A)=\Omega_{A / k}^{p}$ and $\operatorname{HH}_{p}^{(p), / k}(A)=0$ for $i>p$. If $A$ is regular, then one also has $\operatorname{HH}_{p}^{(i), / k}(A)=0$ for all $i<p$.

One can sheafify the construction of the last paragraph. In particular, for any scheme $X / k$, one has a decomposition

$$
\mathcal{H} \mathcal{H}_{p, X}^{/ k} \cong \oplus \mathcal{H} \mathcal{H}_{p, X}^{(i), / k}
$$

If $X$ is smooth, then the only non-vanishing term on the right is the $i=p$ term, where one has $\mathcal{H} \mathcal{H}_{p, X}^{(p), / k}=\Omega_{X / k}^{p}$.

Let $F$ be a subfield of $k$, and let $A$ be a $k$-algebra. Then one has the following spectral sequence:

Lemma 2.10. ([11, 4.3a]) For each $p \geqslant 1$ there is a bounded second quadrant homological spectral sequence $(0 \leqslant i<p, j \geqslant 0)$

$$
{ }_{p} E_{-i, i+j}^{1}=\Omega_{k / F}^{i} \otimes_{k} \mathrm{HH}_{p-i+j}^{(p-i), / k}(A) \longrightarrow \mathrm{HH}_{p+j}^{(p), / F}(A) .
$$

One can sheafify this spectral sequence to obtain a spectral sequence of sheaves on $X$

$$
{ }_{p} E_{-i, i+j}^{1}=\Omega_{k / F}^{i} \otimes_{k} \mathcal{H H}_{p-i+j, X}^{(p-i), / k} \longrightarrow \mathcal{H H}_{p+j, X}^{(p), / F}
$$

Suppose now that $X / k$ is smooth and $Y$ is a closed subvariety of $X$, and $Y_{n}$ is the $n$th infinitesimal neighborhood of $Y$. Then one obtains a spectral sequence of pro-sheaves on $Y$

$$
{ }_{p} E_{-i, i+j}^{1}=\left(\Omega_{k / F}^{i} \otimes_{k} \mathcal{H} \mathcal{H}_{p-i+j, Y_{n}}^{(p-i), / k}\right) \longrightarrow\left(\mathcal{H} \mathcal{H}_{p+j, Y_{n}}^{(p), / F}\right)
$$

Since $X$ is smooth, by Lemma 2.5 and the previous remarks, the only non-zero $E^{1}$ terms are the ones where $j=0$, and, in this case, one has

$$
{ }_{p} E_{-i, i+j}^{1}=\left(\Omega_{k / F}^{i} \otimes_{k} \Omega_{Y_{n} / k}^{p-i}\right) .
$$

In particular, one has has a finite decreasing exhaustive filtration $F^{\cdot}$ of $\left(\Omega_{Y_{n} / F}^{p}\right)$ such that

$$
g r_{F}^{i}:=F^{i} / F^{i+1} \cong\left(\left(\Omega_{k / F}^{i} \otimes_{k} \Omega_{Y_{n} / k}^{p-i}\right)\right) .
$$

Note that $F^{0}=\left(\Omega_{Y_{n} / F}^{p}\right)$ and $F^{p+1}=0$. 


\section{Sheaves of Differentials}

In the following, let $X$ be as before, a smooth projective variety over $k$. We let $Y \subset X$ denote a smooth subvariety, and $Y_{n}$ the $n$th infinitesimal neighborhood of $Y$ in $X$. In particular, if $\mathcal{I}$ is the ideal of definition of $Y$, then $\mathcal{I}^{n+1}$ is the ideal of definition of $Y_{n}$ and $Y=Y_{0}$. Let $\left(\mathfrak{X}, \mathcal{O}_{\mathfrak{X}}\right)$ denote the corresponding formal scheme. Let $\Omega_{X / k}^{p}, \Omega_{Y_{n} / k}^{p}$, and $\Omega_{\mathfrak{X} / k}^{p}$ denote the corresponding sheaves of differential forms. If $F$ is a subfield of $k$, then let $\Omega_{X / F}^{p}, \Omega_{Y_{n} / F}^{p}$, and $\Omega_{\mathfrak{X} / F}^{p}$ denote the corresponding sheaves of differential forms over $F$. Finally, let $\left(\Omega_{X / F}, d\right),\left(\Omega_{Y_{n} / F}, d\right)$, and, $\left(\Omega_{\mathfrak{X} / F}, d\right)$ denote the corresponding de Rham complexes.

\subsection{De Rham cohomology sheaves}

We start by recalling a theorem of Ogus comparing the de Rham cohomology sheaves $\mathcal{H}_{d R}^{*}\left(Y_{n} / F\right)$ and $\mathcal{H}_{d R}^{*}(Y / F)$.

Theorem 3.1. ([12, Theorem 1.3]) The natural restriction map of de Rham complexes

$$
\Omega_{\mathfrak{X} / F} \longrightarrow \Omega_{Y_{n} / F}
$$

is a quasi-isomorphism. In particular, the natural restriction maps

$$
\mathcal{H}_{d R}\left(Y_{n} / F\right) \longrightarrow \mathcal{H}_{d R}(Y / F)
$$

are isomorphisms.

Note that in loc. cit. this result is proved when $X$ is smooth over $k=\mathbb{C}$; however, the same proof applies to any $k$ of characteristic zero. More generally, Ogus's proof also applies to de Rham complexes over subfields $F \subset k$.

\subsection{Continuous cohomology of cotangent sheaves over $k$}

In this section, we assume further that $Y$ is a smooth ample hyperplane section. For any subfield $F \subset k$, one has a natural surjection of sheaves of abelian groups on $Y$

$$
\Omega_{Y_{n} / F}^{1} \longrightarrow \Omega_{Y / F}^{1} \longrightarrow 0 .
$$

Let $\Omega_{\left(Y_{n}, Y\right) / F}^{1}$ denote the kernel of this morphism. Similarly, one has a natural surjection

$$
\Omega_{Y_{n} / F}^{q} \longrightarrow \Omega_{Y / F}^{q} \longrightarrow 0 .
$$

Again, let $\Omega_{\left(Y_{n}, Y\right) / F}^{q}$ denote the kernel. In $[\mathbf{1 3}]$, the authors proved the $q=1$ case of the following theorem.

Theorem 3.2. Let $X$ be a smooth projective variety over $k$, and let $Y \subset X$ be a smooth ample hyperplane section. Then the natural restriction map

$$
\mathrm{H}^{p}\left(Y, \Omega_{Y_{n} / k}^{q}\right) \longrightarrow \mathrm{H}^{p}\left(Y, \Omega_{Y / k}^{q}\right)
$$

is an isomorphism for $p+q<\operatorname{dim}(Y)-1$ and an injection for $p+q=\operatorname{dim}(Y)-1$. In particular,

$$
\mathrm{H}^{p}\left(Y, \Omega_{\left(Y_{n}, Y\right) / k}^{q}\right)=0
$$

for all $p+q<\operatorname{dim}(Y)$. 
Corollary 3.3. Let $X$ and $Y$ be as in the theorem. Then the natural restriction map

$$
\mathrm{H}_{\text {cont }}^{p}\left(Y,\left(\Omega_{Y_{n} / k}^{q}\right)\right) \longrightarrow \mathrm{H}^{p}\left(Y, \Omega_{Y / k}^{q}\right)
$$

is an isomorphism for $p+q<\operatorname{dim}(Y)-1$ and an injection for $p+q=\operatorname{dim}(Y)-1$.

Proof. Recall that one has an exact sequence

$$
0 \longrightarrow R^{1} \varliminf_{\varlimsup} H^{p-1}\left(Y, \Omega_{Y_{n} / k}^{q}\right) \longrightarrow \mathrm{H}_{\text {cont }}^{p}\left(Y,\left(\Omega_{Y_{n} / k}^{q}\right)\right) \longrightarrow \operatorname{limH}^{p}\left(Y, \Omega_{Y_{n} / k}^{q}\right) \longrightarrow 0 .
$$

Theorem 3.2 implies that the pro-system $\left(\mathrm{H}^{p-1}\left(Y, \Omega_{Y_{n} / k}^{q}\right)\right)$ satisfies the Mittag-Leffler condition for all $p+q<\operatorname{dim}(Y)$. Therefore, the left-most term in the above exact sequence is zero for all $p+q<\operatorname{dim}(Y)$. Again, by the previous theorem, one has an isomorphism

$$
\lim ^{p}\left(Y, \Omega_{Y_{n} / k}^{q}\right) \longrightarrow \mathrm{H}^{p}\left(Y, \Omega_{Y / k}^{q}\right)
$$

for $p+q<\operatorname{dim}(Y)-1$. This gives the desired isomorphism for $p+q<\operatorname{dim}(Y)-1$. Suppose now that $p+q=\operatorname{dim}(Y)-1$. In this case, the $R^{1}$ term above still vanishes. Furthermore, since taking inverse limits is left exact, the previous theorem gives an injection

$$
\lim _{\longleftarrow} H^{p}\left(Y, \Omega_{Y_{n} / k}^{q}\right) \longrightarrow \mathrm{H}^{p}\left(Y, \Omega_{Y / k}^{q}\right)
$$

We begin with some preliminary lemmas.

Lemma 3.4. Let $X$ and $Y$ be as in the theorem. For all $q>0$ and $n \geqslant 0$, one has an exact sequence of sheaves on $Y$

$$
0 \longrightarrow \Omega_{Y / k}^{q-1} \otimes_{\mathcal{O}_{X}} \mathcal{I}^{n+1} / \mathcal{I}^{n+2} \longrightarrow \Omega_{X / k}^{q} \otimes_{\mathcal{O}_{X}} \mathcal{O}_{Y_{n}} \longrightarrow \Omega_{Y_{n} / k}^{q} \longrightarrow 0 .
$$

Proof. By Lemma 4.2 in [13], one has the following cotangent sheaf sequence:

$$
0 \rightarrow \mathcal{I}^{n+1} / \mathcal{I}^{n+2} \rightarrow \Omega_{X / k}^{1} \otimes_{\mathcal{O}_{X}} \mathcal{O}_{Y_{n}} \rightarrow \Omega_{Y_{n} / k}^{1} \rightarrow 0 .
$$

Lemma 18.4 in [14], (applied twice; the second time with a suitable twist) gives rise to a four-term sequence

$$
\Omega_{X / k}^{q-2} \otimes_{\mathcal{O}_{X}} \mathcal{I}^{n+2} / \mathcal{I}^{n+3} \longrightarrow \Omega_{X / k}^{q-1} \otimes_{\mathcal{O}_{X}} \mathcal{I}^{n+1} /\left.\mathcal{I}^{n+2} \longrightarrow \Omega_{X / k}^{q}\right|_{Y_{n}} \longrightarrow \Omega_{Y_{n} / k}^{q} \longrightarrow 0 .
$$

By op. cit. this sequence is exact everywhere except perhaps at the second term, where one first directly checks that the composite of the first and second maps (from the left) is zero.

Further, when $n=0$, the cokernel of the first map above can be computed, so that we get the exact sequences (see $[\mathbf{9}, 5.16$ (d), p. 126])

$$
\begin{gathered}
0 \rightarrow \mathcal{I} / \mathcal{I}^{2} \otimes_{\mathcal{O}_{X}} \Omega_{Y / k}^{q-1} \rightarrow \Omega_{X / k}^{q} \otimes_{\mathcal{O}_{X}} \mathcal{O}_{Y} \rightarrow \Omega_{Y / k}^{q} \rightarrow 0 \text { and } \\
\mathcal{I}^{n+2} / \mathcal{I}^{n+3} \otimes_{\mathcal{O}_{X}} \Omega_{X / k}^{q-2} \rightarrow \Omega_{X / k}^{q-1} \otimes_{\mathcal{O}_{X}} \mathcal{I}^{n+1} / \mathcal{I}^{n+2} \rightarrow \Omega_{Y / k}^{q-1} \otimes \mathcal{I}^{n+1} / \mathcal{I}^{n+2} \rightarrow 0 .
\end{gathered}
$$

Putting (5) and (3) together, we get an exact sequence

$$
\Omega_{Y / k}^{q-1} \otimes_{\mathcal{O}_{X}} \mathcal{I}^{n+1} / \mathcal{I}^{n+2} \longrightarrow \Omega_{X / k}^{q} \otimes_{\mathcal{O}_{X}} \mathcal{O}_{Y_{n}} \longrightarrow \Omega_{Y_{n} / k}^{q} \longrightarrow 0 .
$$

Now all that remains to be checked is left exactness in the above sequence. This is 
done by noting that the composite

$$
\Omega_{Y / k}^{q-1} \otimes_{\mathcal{O}_{X}} \mathcal{I}^{n+1} / \mathcal{I}^{n+2} \longrightarrow \Omega_{X / k}^{q} \otimes_{\mathcal{O}_{X}} \mathcal{O}_{Y_{n}} \longrightarrow \Omega_{X / k}^{q} \otimes_{\mathcal{O}_{X}} \mathcal{O}_{Y}
$$

is the same as the composite of the injective maps

$$
\Omega_{Y / k}^{q-1} \otimes_{\mathcal{O}_{X}} \mathcal{I}^{n+1} / \mathcal{I}^{n+2} \longrightarrow \Omega_{X / k}^{q} \otimes_{\mathcal{O}_{X}} \mathcal{I}^{n} / \mathcal{I}^{n+1} \longrightarrow \Omega_{X / k}^{q} \otimes_{\mathcal{O}_{X}} \mathcal{O}_{Y}
$$

(the map on the left is obtained by tensoring the injective map in (4) with the locally free sheaf $\mathcal{I}^{n} / \mathcal{I}^{n+1}$ and hence is injective). This proves the desired left exactness.

Remark 3.5. Alternatively, as pointed out by the referee, the previous lemma can also be proved by first noting that the statement is local in the etale topology on $X$, and therefore one is reduced to $Y=\mathbb{A}^{d-1}$ and $X=\mathbb{A}^{d}$, where the statement is clear.

Lemma 3.6. Let $X$ and $Y$ be as above. Then

$$
\mathrm{H}^{p}\left(Y,\left.\Omega_{X / k}^{q}\right|_{Y} \otimes_{\mathcal{O}_{X}} \mathcal{I}^{n} / \mathcal{I}^{n+1}\right)=0
$$

for all $0 \leqslant p+q<\operatorname{dim}(Y)$.

Proof. We have an exact sequence

$$
0 \longrightarrow \Omega_{Y / k}^{q-1} \otimes \mathcal{I} /\left.\mathcal{I}^{2} \longrightarrow \Omega_{X / k}^{q}\right|_{Y} \rightarrow \Omega_{Y / k}^{q} \longrightarrow 0 .
$$

Tensoring the above exact sequence with the locally free sheaf $\mathcal{I}^{n} / \mathcal{I}^{n+1}$ gives an exact sequence

$$
0 \longrightarrow \Omega_{Y / k}^{q-1} \otimes \mathcal{I} / \mathcal{I}^{2} \otimes \mathcal{I}^{n} /\left.\mathcal{I}^{n+1} \longrightarrow \Omega_{X / k}^{q}\right|_{Y} \otimes \mathcal{I}^{n} / \mathcal{I}^{n+1} \rightarrow \Omega_{Y / k}^{q} \otimes \mathcal{I}^{n} / \mathcal{I}^{n+1} \longrightarrow 0
$$

Then, by Kodaira-Nakano vanishing, one has that $\mathrm{H}^{p}\left(Y, \Omega_{Y / k}^{q-1} \otimes \mathcal{I} / \mathcal{I}^{2} \otimes \mathcal{I}^{n} / \mathcal{I}^{n+1}\right)=$ 0 for all $p+q-1<\operatorname{dim}(Y)$. It follows that the cohomology of the middle term and the right-most term are isomorphic for all $p+q<\operatorname{dim}(Y)$. On the other hand, Kodaira-Nakano vanishing applied to the right-most term gives that $\mathrm{H}^{p}\left(Y, \Omega_{Y / k}^{q} \otimes\right.$ $\left.\mathcal{I}^{n} / \mathcal{I}^{n+1}\right)=0$ for all $p+q<\operatorname{dim}(Y)$. Therefore, $\mathrm{H}^{p}\left(Y, \Omega_{X / k}^{q} \otimes \mathcal{I}^{n} / \mathcal{I}^{n+1}\right)=0$ for all $p+q<\operatorname{dim}(Y)$.

Proof. (Theorem 3.2) Consider the following commutative diagram of sheaves on $Y$ :

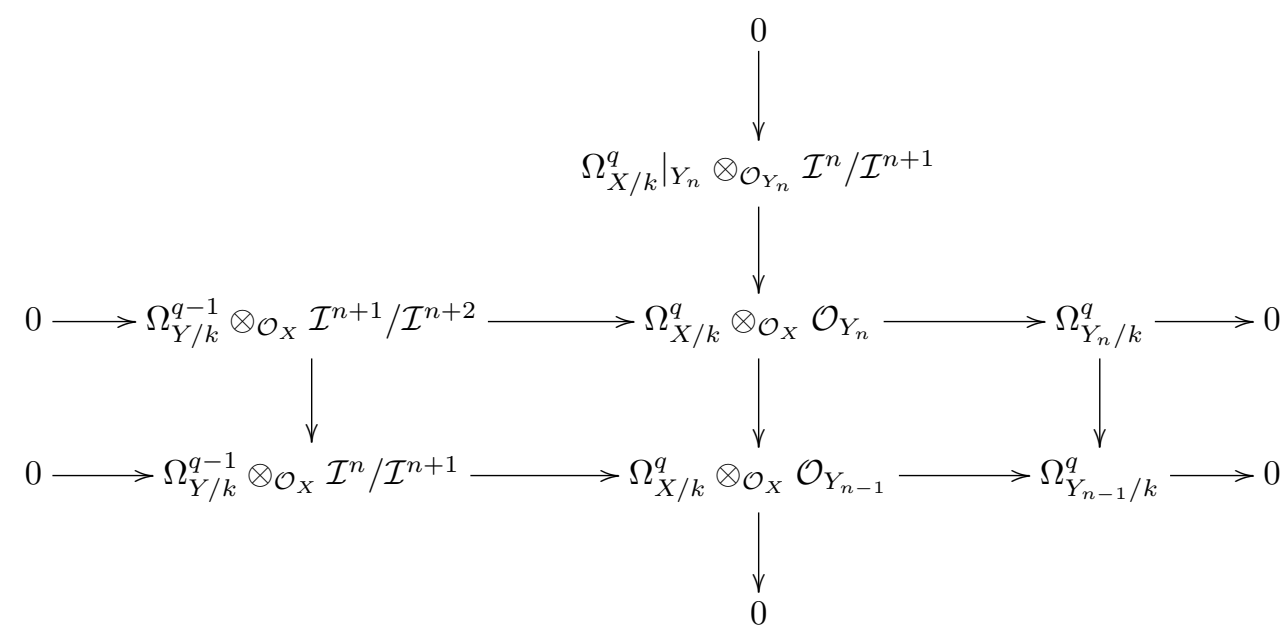


Here the left-most vertical arrow is zero. The bottom two rows are exact by Lemma 3.4. The middle column is given by tensoring the standard exact sequence

$$
0 \longrightarrow \mathcal{I}^{n} / \mathcal{I}^{n+1} \longrightarrow \mathcal{O}_{Y_{n}} \longrightarrow \mathcal{O}_{Y_{n-1}} \longrightarrow 0
$$

with the locally free sheaf $\left.\Omega_{X / k}^{p}\right|_{Y_{n}}$ and is therefore also exact. By Lemma 3.6,

$$
\mathrm{H}^{p}\left(Y,\left.\Omega_{X}^{q}\right|_{Y} \otimes \mathcal{I}^{n} / \mathcal{I}^{n+1}\right)=0
$$

for all $p+q<\operatorname{dim}(Y)$. Therefore, taking the long exact sequence in cohomology associated to the middle vertical column in the diagram above gives isomorphisms

$$
\mathrm{H}^{p}\left(Y, \Omega_{X / k}^{q} \otimes_{\mathcal{O}_{X}} \mathcal{O}_{Y_{n}}\right) \longrightarrow \mathrm{H}^{p}\left(Y, \Omega_{X / k}^{q} \otimes_{\mathcal{O}_{X}} \mathcal{O}_{Y_{n-1}}\right)
$$

for all $p+q<\operatorname{dim}(Y)-1$ and an injection when $p+q=\operatorname{dim}(Y)-1$. Taking cohomology of the horizontal exact sequences in the diagram above gives the following diagram of long exact sequences:

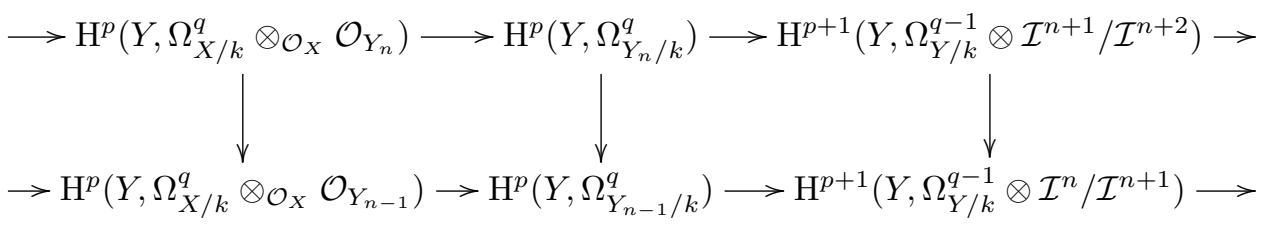

By the previous remarks and Lemma 3.6, it follows that the restriction map

$$
\mathrm{H}^{p}\left(Y, \Omega_{Y_{n} / k}^{q}\right) \longrightarrow \mathrm{H}^{p}\left(Y, \Omega_{Y_{n-1} / k}^{q}\right)
$$

is an isomorphism when $p+q<\operatorname{dim}(Y)-1$ and an injection when $p+q=\operatorname{dim}(Y)-1$. In particular, the restriction morphism

$$
\mathrm{H}^{p}\left(Y, \Omega_{Y_{n} / k}^{q}\right) \longrightarrow \mathrm{H}^{p}\left(Y, \Omega_{Y / k}^{q}\right)
$$

is an isomorphism when $p+q<\operatorname{dim}(Y)-1$, and an injection when $p+q=\operatorname{dim}(Y)-1$.

Finally, applying this result to the long exact sequence in cohomology associated to the short exact sequence

$$
0 \longrightarrow \Omega_{\left(Y_{n}, Y\right) / k}^{q} \longrightarrow \Omega_{Y_{n} / k}^{q} \longrightarrow \Omega_{Y / k}^{q} \longrightarrow 0
$$

now gives the second part of the theorem.

In the rest of this section, we use the previous results to investigate the following morphism:

$$
\mathrm{H}_{\text {cont }}^{p}\left(Y,\left(\Omega_{Y_{n} / k}^{q} / d \Omega_{Y_{n} / k}^{q-1}\right)\right) \longrightarrow \mathrm{H}^{p}\left(Y, \Omega_{Y / k}^{q} / d \Omega_{Y / k}^{q-1}\right) .
$$

In particular, we will prove the following theorem:

Theorem 3.7. Let $X$ and $Y$ be as in Theorem 3.2 and suppose that $q \geqslant 1$. Then the natural restriction morphism

$$
\mathrm{H}_{\text {cont }}^{p}\left(Y,\left(\Omega_{Y_{n} / k}^{q} / d \Omega_{Y_{n} / k}^{q-1}\right)\right) \longrightarrow \mathrm{H}^{p}\left(Y, \Omega_{Y / k}^{q} / d \Omega_{Y / k}^{q-1}\right) .
$$

is an isomorphism for all $p+q<\operatorname{dim}(Y)-1$ and an injection for $p+q=\operatorname{dim}(Y)-1$. 
We begin with some preliminary remarks. First, note that we have a diagram of pro-sheaves:

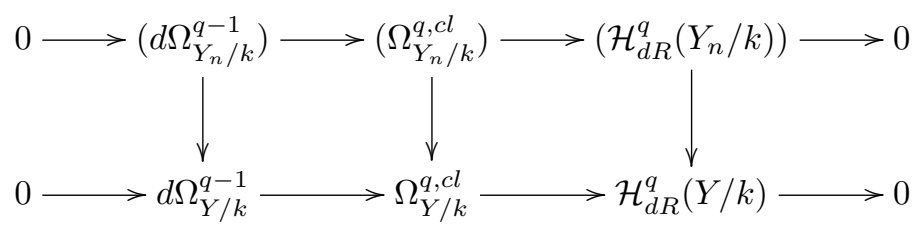

Here $\Omega_{Y_{n} / k}^{p, c l}$ denotes the sheaf of (locally) closed forms and $\mathcal{H}_{d R}^{q}\left(Y_{n} / k\right)$ are the usual de Rham cohomology sheaves. In particular, the rows in the above commutative diagram are exact by definition. Furthermore, by Theorem 3.1, the pro-systems $\left(\mathcal{H}_{d R}^{q}\left(Y_{n} / k\right)\right)$ are constant and, in particular, isomorphic to the constant pro-system $\left(\mathcal{H}_{d R}^{q}(Y / k)\right)$. It follows that one has isomorphisms

$$
\mathrm{H}_{\text {cont }}^{p}\left(Y,\left(\mathcal{H}_{d R}^{q}\left(Y_{n} / k\right)\right)\right) \longrightarrow \mathrm{H}^{p}\left(Y, \mathcal{H}_{d R}^{q}(Y / k)\right) .
$$

Proposition 3.8. The natural morphism

$$
\mathrm{H}_{\text {cont }}^{p}\left(Y,\left(d \Omega_{Y_{n} / k}^{q-1}\right)\right) \longrightarrow \mathrm{H}^{p}\left(Y, d \Omega_{Y / k}^{q-1}\right)
$$

is an isomorphism for $p+q<N$ and an injection for $p+q=N$ if and only if the morphism

$$
\mathrm{H}_{\text {cont }}^{p}\left(Y,\left(\Omega_{Y_{n} / k}^{q, c l}\right)\right) \longrightarrow \mathrm{H}^{p}\left(Y, \Omega_{Y / k}^{q, c l}\right)
$$

is an isomorphism for $p+q<N$ and an injection for $p+q=N$.

Proof. The commutative diagram above induces the following diagram in cohomology where the rows are exact:

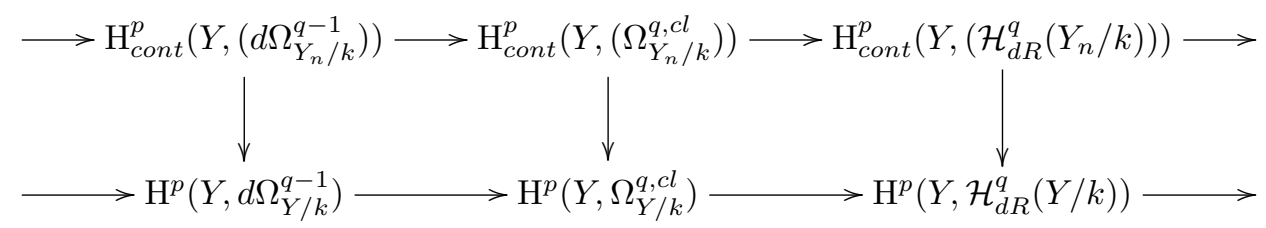

The result is now a consequence of the 5-lemma and the fact that the restriction maps

$$
\mathrm{H}_{\text {cont }}^{p}\left(Y,\left(\mathcal{H}_{d R}^{q}\left(Y_{n} / k\right)\right)\right) \longrightarrow \mathrm{H}^{p}\left(Y, \mathcal{H}_{d R}^{q}(Y / k)\right)
$$

are isomorphisms.

Proof. (Theorem 3.7) Consider the following two statements:

(1) The following morphism is an isomorphism for all $p+q<\operatorname{dim}(Y)-1$ and injective for $p+q=\operatorname{dim}(Y)-1$ :

$$
\mathrm{H}_{\text {cont }}^{p}\left(Y,\left(\Omega_{Y_{n} / k}^{q} / d \Omega_{Y_{n} / k}^{q-1}\right)\right) \longrightarrow \mathrm{H}^{p}\left(Y, \Omega_{Y / k}^{q} / d \Omega_{Y / k}^{q-1}\right) .
$$

(2) The following morphism is an isomorphism for all $p+q<\operatorname{dim}(Y)-1$ and injective for $p+q=\operatorname{dim}(Y)-1$ :

$$
\mathrm{H}_{\text {cont }}^{p}\left(Y,\left(d \Omega_{Y_{n} / k}^{q}\right)\right) \longrightarrow \mathrm{H}^{p}\left(Y, d \Omega_{Y / k}^{q}\right) .
$$


We shall prove both of these statements simultaneously via induction on $q$. We begin with the base case $q=1$. Consider the following commutative diagram with exact rows:

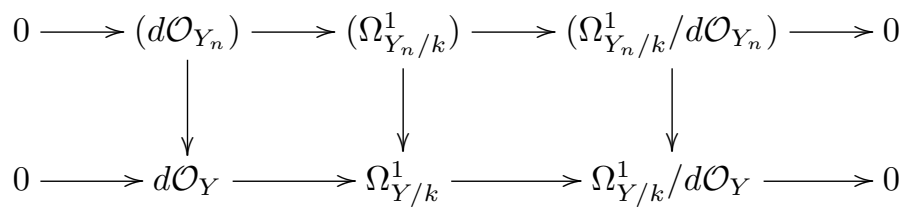

By considering the diagram of long exact sequences in cohomology associated to the above diagram and applying Corollary 3.3, one concludes that statement (1) for $q=1$ follows if the natural restriction map

$$
\mathrm{H}_{\text {cont }}^{p}\left(Y,\left(d \mathcal{O}_{Y_{n}}\right)\right) \longrightarrow \mathrm{H}^{p}\left(Y, d \mathcal{O}_{Y}\right)
$$

is an isomorphism for all $p<\operatorname{dim}(Y)-1$ and an injection for $p=\operatorname{dim}(Y)-1$. On the other hand, we have a commutative diagram with exact rows

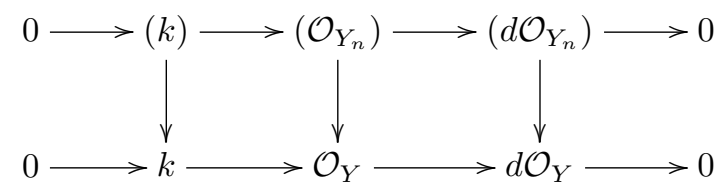

Therefore,

$$
\mathrm{H}_{\text {cont }}^{p}\left(Y,\left(d \mathcal{O}_{Y_{n}}\right)\right) \longrightarrow \mathrm{H}^{p}\left(Y, d \mathcal{O}_{Y}\right)
$$

is an isomorphism if and only if

$$
\mathrm{H}_{\text {cont }}^{p}\left(Y,\left(\mathcal{O}_{Y_{n}}\right)\right) \longrightarrow \mathrm{H}^{p}\left(Y, \mathcal{O}_{Y}\right)
$$

is an isomorphism. The result now follows from the exact sequences

$$
0 \longrightarrow \mathcal{I} / \mathcal{I}^{n+1} \longrightarrow \mathcal{O}_{Y_{n}} \longrightarrow \mathcal{O}_{Y} \longrightarrow 0
$$

and Kodaira-Nakano vanishing. This proves statement (1) for $q=1$. Now for statement (2), in the case $q=1$, consider the following diagram with exact rows:

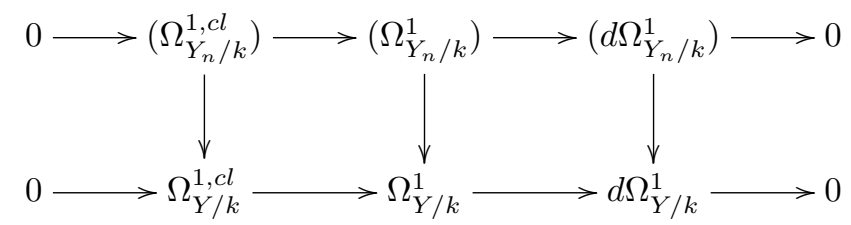

Just as above, it is enough to show that the restriction map

$$
\mathrm{H}_{\text {cont }}^{p}\left(Y,\left(\Omega_{Y_{n} / k}^{1, c l}\right)\right) \longrightarrow \mathrm{H}^{p}\left(Y, \Omega_{Y / k}^{1, c l}\right)
$$

is an isomorphism for $p<\operatorname{dim}(Y)-1$ and an injection for $p=\operatorname{dim}(Y)-1$. By Proposition 3.8, this is equivalent to showing that the restriction map

$$
\mathrm{H}_{\text {cont }}^{p}\left(Y,\left(d \mathcal{O}_{Y_{n}}\right)\right) \longrightarrow \mathrm{H}^{1}\left(Y, d \mathcal{O}_{Y}\right)
$$

is an isomorphism for all $p<\operatorname{dim}(Y)-1$ and an injection for $p=\operatorname{dim}(Y)-1$. We have already seen this above. 
Suppose that the statements hold for all fixed $i<q$. First, note that we have a commutative diagram with exact rows

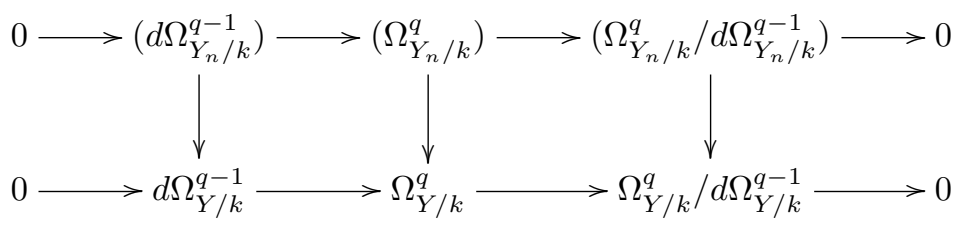

This gives the following commutative diagram with exact rows:

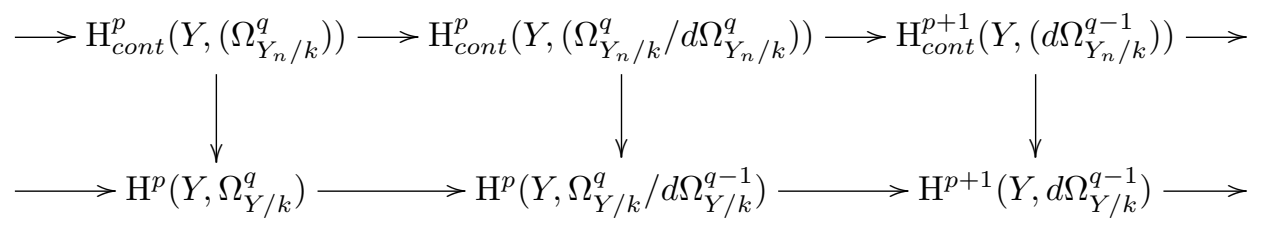

Therefore, statement (1) follows from the induction hypothesis, Corollary 3.3, and a diagram chase. It remains to prove statement (2). Now consider the following commutative diagram with exact rows:

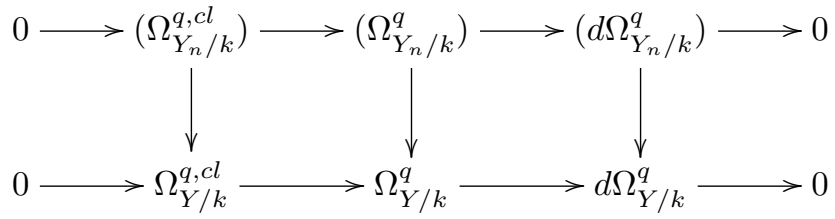

This gives the following diagram in cohomology:

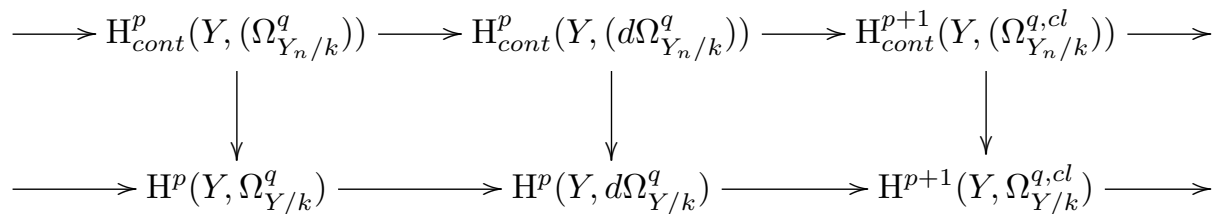

Once again, the result follows from an application of Corollary 3.3, Proposition 3.8, and a diagram chase.

\subsection{Continuous cohomology of cotangent sheaves over $F$}

We use the same notation and hypothesis as in the previous section. In particular, $X$ is a smooth projective variety over $k$ and $F \subset k$ is a fixed subfield. In this section, we generalize the main theorems of the previous section to similar results for differentials relative to $F$. These results will be used in the next section to compute cyclic homology relative to $F=\mathbb{Q}$.

Theorem 3.9. Let $X$ and $Y$ be as in the theorem. Then the natural restriction map

$$
\mathrm{H}_{\text {cont }}^{p}\left(Y,\left(\Omega_{Y_{n} / F}^{q}\right)\right) \longrightarrow \mathrm{H}^{p}\left(Y, \Omega_{Y / F}^{q}\right)
$$

is an isomorphism for all $p+q<\operatorname{dim}(Y)-1$ and an injection for $p+q=\operatorname{dim}(Y)-1$. 
Proof. The Kassel-Sletsjøe spectral sequence (cf. §2.4) gives a filtration $F^{*}$ of $\left(\Omega_{Y_{n} / F}^{q}\right)$ such that

$$
g r_{F}^{i}:=F^{i} / F^{i+1} \cong\left(\left(\Omega_{k / F}^{i} \otimes_{F} \Omega_{Y_{n} / k}^{q-i}\right)\right)
$$

$F^{0}=\left(\Omega_{Y_{n} / F}^{q}\right)$, and $F^{q+1}=0$. Recall that if $V$ is a finite-dimensional vector space over $k$, then one has an isomorphism

$$
\mathrm{H}^{p}\left(Y, V \otimes_{k} \mathcal{F}\right) \cong \mathrm{H}^{p}(Y, \mathcal{F}) \otimes_{k} V .
$$

Filtering an arbitrary vector space by finite-dimensional subspaces and using that cohomology commutes with direct limits gives the same result for arbitrary $V$. In particular, one has isomorphisms

$$
\mathrm{H}^{p}\left(Y, \Omega_{k / F}^{i} \otimes_{k} \Omega_{Y / k}^{q-i}\right) \cong \mathrm{H}^{p}\left(Y, \Omega_{Y / k}^{q-i}\right) \otimes_{k} \Omega_{k / F}^{i} .
$$

One has a similar result for continuous cohomology. Therefore, the result now follows by recurrence, using the filtration above, and the fact that it holds over $k$. We leave the details to the reader.

Theorem 3.10. Let $X$ and $Y$ be as above, and suppose that $1 \leqslant q$. Then the natural restriction morphism

$$
\mathrm{H}_{\text {cont }}^{p}\left(Y,\left(\Omega_{Y_{n} / F}^{q} / d \Omega_{Y_{n} / F}^{q-1}\right)\right) \longrightarrow \mathrm{H}^{p}\left(Y, \Omega_{Y / F}^{q} / d \Omega_{Y / F}^{q-1}\right)
$$

is an isomorphism for all $p+q<\operatorname{dim}(Y)-1$ and an injection for $p+q=\operatorname{dim}(Y)-1$.

Proof. Given the previous theorem, and Theorem 3.1, the proof of this theorem is exactly the same as that of Theorem 3.7. Note that Proposition 3.8 also holds over $F$.

\section{Main Theorem}

As in the previous sections, we let $X$ denote a smooth projective variety over $k$, and $Y \subset X$ is a smooth ample hyperplane section. Let $Y_{n}$ denote the $n$th infinitesimal thickening of $Y$ in $X$.

Theorem 4.1. The natural restriction map

$$
\mathrm{H}_{\text {cont }}^{p}\left(Y,\left(\mathcal{K}_{q, Y_{n}}\right)\right) \longrightarrow \mathrm{H}^{p}\left(Y, \mathcal{K}_{q, Y}\right)
$$

is an isomorphism for all $p+q<\operatorname{dim}(Y)$ and injective for $p+q=\operatorname{dim}(Y)$.

Proof. Recall that we have an exact sequence of pro-sheaves

$$
0 \longrightarrow\left(\mathcal{K}_{q,\left(Y_{n}, Y\right)}\right) \longrightarrow\left(\mathcal{K}_{\left.q, Y_{n}\right)}\right) \longrightarrow \mathcal{K}_{q, Y} \longrightarrow 0 .
$$

Therefore, it is enough to show that

$$
\mathrm{H}_{\text {cont }}^{p}\left(Y,\left(\mathcal{K}_{q,\left(Y_{n}, Y\right)}\right)\right)=0
$$

for all $p+q<\operatorname{dim}(Y)+1$. By Corollary 2.9 , it is enough to show that

$$
\mathrm{H}_{\text {cont }}^{p}\left(Y,\left(\mathcal{H C}_{q-1,\left(Y_{n}, Y\right)}^{/ \mathbb{Q}}\right)\right)=0
$$

for $p+q<\operatorname{dim}(Y)+1$. For the latter, it is enough to show that the natural restriction 
maps

$$
\mathrm{H}_{\text {cont }}^{p}\left(Y,\left(\mathcal{H C}_{q-1, Y_{n}}^{/ \mathbb{Q}}\right)\right) \longrightarrow \mathrm{H}^{p}\left(Y, \mathcal{H C}_{q-1, Y}^{/ \mathbb{Q}}\right)
$$

is an isomorphism for $p+q<\operatorname{dim}(Y)$ and an injection for $p+q=\operatorname{dim}(Y)$. On the other hand, by Corollary 2.6

$$
\left(\mathcal{H C}_{q, Y_{n}}^{/ \mathbb{Q}}\right) \cong\left(\Omega_{Y_{n} / \mathbb{Q}}^{q} / d \Omega_{Y_{n} / \mathbb{Q}}^{q-1}\right) \oplus\left(\mathcal{H}_{d R}^{q-2}\left(Y_{n} / \mathbb{Q}\right)\right) \oplus\left(\mathcal{H}_{d R}^{q-4}\left(Y_{n} / \mathbb{Q}\right)\right) \cdots
$$

The result now follows from Theorem 3.10 and Theorem 3.1.

Corollary 4.2. The natural restriction map

$$
\mathrm{H}_{\text {cont }}^{p}\left(\mathfrak{X},\left(\mathcal{K}_{p, Y_{n}}\right)\right) \longrightarrow \mathrm{H}^{p}\left(Y, \mathcal{K}_{p, Y}\right)
$$

is an isomorphism for all $p<\operatorname{dim}(Y) / 2$.

\section{References}

[1] M. Artin and B. Mazur, Etale homotopy, volume 100 of Lecture Notes in Mathematics, Springer-Verlag, Berlin, 1986 (reprint of the 1969 original).

[2] S. Bloch, $K_{2}$ of Artinian $Q$-algebras, with application to algebraic cycles, Comm. Algebra 3 (1975), 405-428.

[3] S. Bloch, H. Esnault, and M. Kerz, p-adic deformations of algebraic cycles, Invent. Math. 195 (2014), no. 3, 673-722.

[4] G. Cortiñas, C. Haesemeyer, and C. Weibel, Infinitesimal cohomology and the Chern character to negative cyclic homology, Math. Ann. 344 (2009), no. 4, 891-922.

[5] T.G. Goodwillie, Cyclic homology, derivations, and the free loopspace, Topology 24 (1985), 187-215.

[6] T.G. Goodwillie, Relative algebraic K-theory and cyclic homology, Ann. of Math. (2) 124 (1986), 347-402.

[7] M. Green and P. Griffiths, Formal deformation of Chow groups, in The Legacy of Niels Henrick Abel, 467-509, Springer, Berlin, 2004.

[8] A. Grothendieck, Cohomologie locale des faisceaux cohérents et théorèmes de Lefschetz locaux et globaux, Advanced Studies in Pure Mathematics, Vol. 2. North-Holland Publishing Co., Amsterdam; Masson \& Cie, Éditeur, Paris, 1968.

[9] R. Hartshorne, Algebraic geometry, Graduate Texts in Mathematics, No. 52, Springer-Verlag, 1977.

[10] U. Jannsen, Continuous étale cohomology, Math. Ann. 280 (1988), 207-245.

[11] C. Kassel and A.B. Sletsjøe, Base change, transitivity and Künneth formulas for the Quillen decomposition of Hochschild homology, Math. Scand. 70 (1992), 186-192.

[12] A. Ogus, The formal Hodge filtration, Invent. Math. 31 (1975/76), 193-228.

[13] D. Patel and G.V. Ravindra, Towards connectivity for codimension two cycles: Infinitesimal deformations, Journal of Algebra 399 (2014), 407-422. 
[14] Stacks Project, http://stacks.math.columbia.edu/download/modules.pdf.

[15] R.W. Thomason and T. Trobaugh, Higher algebraic K-theory of schemes and of derived categories, in The Grothendieck Festschrift, Vol. III, 247-435, Progr. Math., 88, Birkhäuser Boston, Boston, MA, 1990.

[16] C. Weibel, An introduction to homological algebra, Cambridge Studies in Advanced Mathematics, 38. Cambridge University Press, Cambridge, 1994.

[17] C. Weibel and S. Geller, Étale descent for Hochschild and cyclic homology, Comment. Math. Helv. 66 (1991), 368-388.

\section{Patel deeppatel1981@gmail.com}

Department of Mathematics, Purdue University, 150 N. University Street, West Lafayette, IN 47907, USA

G. V. Ravindra girivarur@umsl.edu

Department of Mathematics and Computer Science, University of Missouri-St. Louis, One University Boulevard, St. Louis, MO 63121, USA 